\title{
HOW CAN HUMANS LEARN FROM COMPUTERS?
}

\author{
Search and Knowledge for Human and Machine Problem Solving \\ Matej Guid \\ PhD thesis, University of Ljubljana, 2010 \\ Reviewed by Dap Hartmann
}

In his PhD thesis, FIDE Chess Master Matej Guid combines three interrelated research endeavors: (1) Measuring human problem solving performance; (2) Improving human problem solving performance; (3) Improving the understanding of heuristic search in computer game playing. The scope of this research project is limited to human and computer game playing, and chess was chosen as the experimental domain. A logical choice when you are a FIDE Master and Ivan Bratko is your thesis supervisor.

To answer the question How can we develop methods based on computer heuristic search for evaluating human problem solving performance? Guid carried out a computer analysis of the (problem solving) performance of World Chess Champions. Every game played in every (classical) World Championship match between 1886 (Steinitz - Zukertort) and 2006 (Kramnik - Topalov) was analyzed by CRAFTY (starting at move 12 and using no opening book) to a depth (iteratively) of 12 ply (plus quiescence search). Under these conditions, it is doubtful whether CRAFTY performs at a World Champion level. Guid discusses the credibility of a heuristic search based estimator and provides a probabilistic model of ranking by 'an imperfect referee' to demonstrate that this does not pose a problem. So, the imperfect referee CRAFTY serves as a solid benchmark against which all former human World Champions were assessed. The following data were collected for each search depth (from 2 to 12 ply): the best move (and score), the second-best move (and score), and the score of the move that was actually played in the game. These data were fed into an algorithm that used various criteria to decide who was the greatest Chess World Champion between 1886 and 2006. The most important criterion was the average difference in the evaluation values between the moves actually played and the best moves found by CRAFTY. This method is somewhat biased and favors positional players over tactical players. The winner is José Raúl Capablanca (World Champion from 1921-1927), followed by Vladimir Kramnik (20002007). Wilhelm Steinitz came in last while Bobby Fischer, rather surprising perhaps, is in $11^{\text {th }}$ place (out of the 14 World Champions). Guid also presents a few other metrics to rank these former World Champions, such as blunder rates and percentage of best moves played. Cuba will undoubtedly be delighted by the outcome of this experiment. However, Guid shows that, on average, Capablanca was faced with 'much easier' positions than Steinitz. The metric that Guid developed to determine the difficulty of a position confirms the established view that "Capablanca is renowned for playing a 'simple' chess and avoiding complications, while it is common that Steinitz and Tal faced many 'wild' positions in their games."

Next, Guid addresses the question How can we develop methods based on computer heuristic search for improving human problem solving? A notoriously difficult question that has puzzled AI researchers for a very long time. As is so often the case, the game of chess serves as the perfect drosophila to test various theories and methods. Guid focuses on computer annotations of chess games. In its simplest form, annotations distinguish between good moves and bad moves. It is relatively easy to point out a bad move by showing that there is a better move. Guid's approach is more refined in that he does not focus on the single value that the evaluation function produces. Instead, he considers individual components (characteristics) that make up the evaluation function: "most moves in games, pitting two players with opposing interests against each other, are a sort of tradeoff of positive and negative characteristics of the position. With most moves you gain something and you lose something." For example, in the game Kasparov-Shirov (Horgen 1994) Shirov played 16. ... Nc5. At this point CRAFTY, equipped with Guid's annotation software, comments: “16. ... Ra7 leads to equality after 17. $\mathrm{Bd} 3 \mathrm{O}-\mathrm{O}$ 18. O-O [...] Black has improved the pawn structure and the activity of his pieces, White no longer has a strong knight. Black no longer has the advantage of a bishop pair." Having made the case that an expert computer annotator is a good way to teach human chess players to improve their game (improve human problem solving in the domain of chess), Guid next discusses what type of knowledge is required for an expert annotator. As a practical case he discusses (in the following chapter) deriving concepts and strategies from chess tablebases. The challenge is to construct human-friendly instructions on how to win such endgames. Even though the approach is admirable and certainly deserve further attention, the results to date are a bit disappointing considering that the method has only been applied to the KBNK endgame.

In the final section of his thesis, Guid analyses the properties of successful evaluation functions (they should exhibit the 'monotonicity principle', which provides the program with a sense of direction towards a goal) and 
he studies factors that influence the behavior of diminished returns with increased search effort. His 'go-deep' experiments on more than 40,000 positions comprise the most extensive research into this phenomenon to date. While the quality of the evaluation function clearly influences the rate of change in the computer's choice of the best move, the intriguing question is: can this finding also be used the other way around? In the final paragraph of his thesis, Matej Guid leaves us with precisely that interesting open question: Could the rates of changed decisions that arise from search to different depths be used as a direct measure of the quality of an evaluation function?

One thing which I particularly like about this thesis, is that many of the questions that came up while I was reading it were addressed later on. Questions such as 'what if you used a stronger chess program than CRAFTY?' (see section 3.6), or 'what about extracting from endgame databases knowledge that can improve the performance of humans chess players?' (see chapter 7) were anticipated and adequately dealt with.

It is good to see that much of Guid's work was already published in the ICGA Journal, and in the conference proceedings series Computer and Games and Advances in Computer Games. Clearly, he has proactively invited an open discussion about his ideas with the computer game-playing community. And he has received useful feedback (if only from referees reports) to further develop his research, which has now accumulated into the present thesis. I wholeheartedly encourage everyone who has progressed to this point in this review to download Matej Guid's very interesting and highly readable thesis from http://eprints.fri.unilj.si/1113/1/Matej_Guid.disertacija.pdf If you find it too much trouble to copy that address into your web browser by hand, simply have Google search for the title of the thesis. It should come up as the first hit. Toplo priporočam - Warmly recommended!

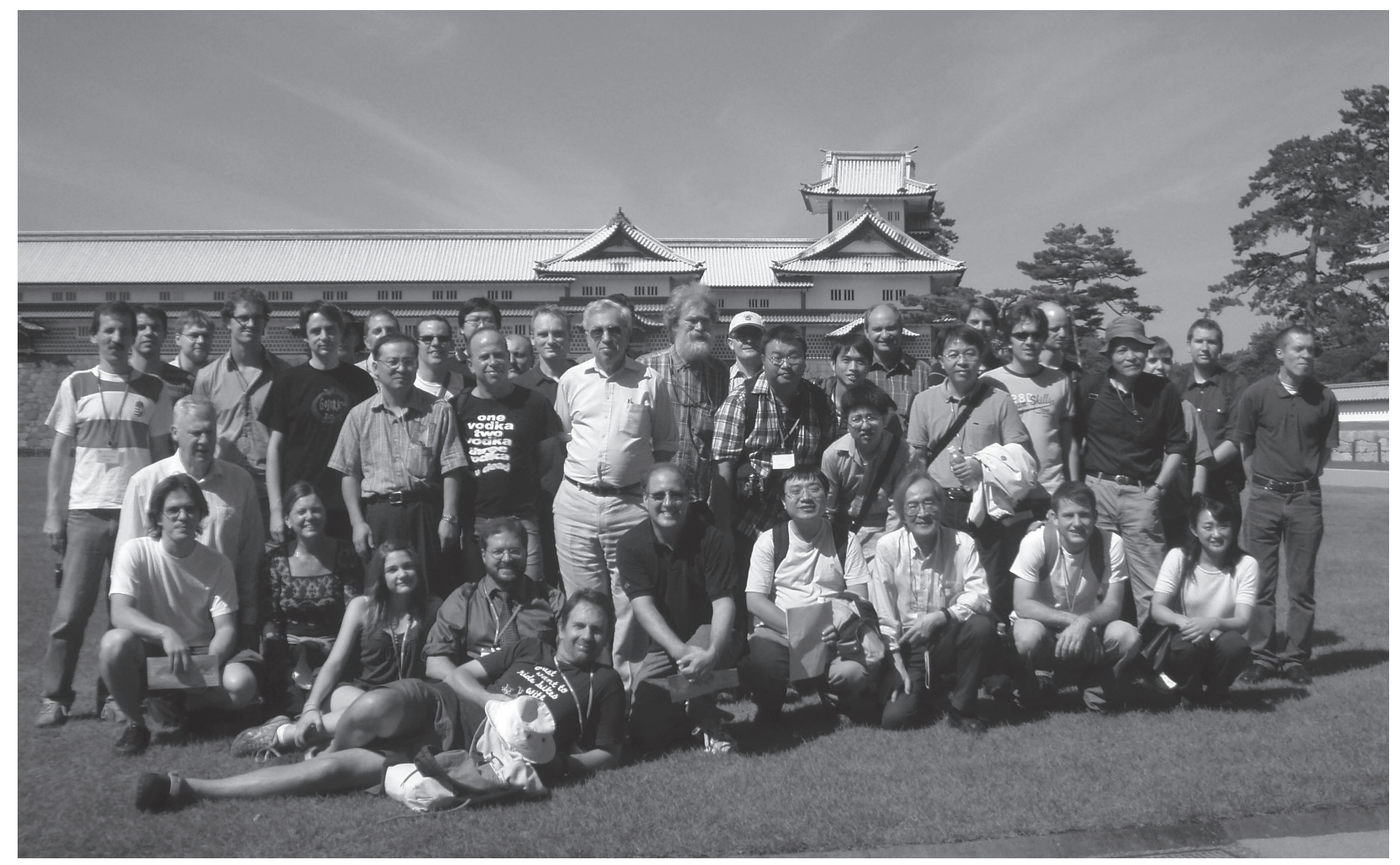

In front of the Kanazawa Castle, Kanazawa, Japan. 